\title{
Intracystic papillary carcinoma of breast- A rare disease entity in males
}

\author{
Afnan Gul ${ }^{1, *}$, Sandhyalakshmi B.N ${ }^{2}$, Srinivasa Murthy $\mathbf{V}^{3}$ \\ ${ }^{1}$ Post Graduate Student, ${ }^{2}$ Assistant Professor, ${ }^{3}$ Director and Professor, Dept. of Pathology, ESIC MC \& PGIMSR Rajajinagar, \\ Bengaluru, Karnataka, India \\ *Corresponding Author: \\ Email: afr.afr.on.the.wall@gmail.com
}

Received: $25^{\text {th }}$ February, 2017

Accepted: $8^{\text {th }}$ December, 2017

\begin{abstract}
Carcinoma of breast is rare in men with an estimated incidence of one per 100,000 per annum. Of all the histological types of breast cancer, Intra-cystic papillary carcinoma (IPC) of the breast is rare accounting for less than $1 \%$ of all breast malignancies. A 61 year old gentleman presented with painless, progressively enlarging lump in his left breast since 3 years associated with serosanginous nipple discharge of 3 months duration. On examination, a single $8 \times 3 \mathrm{~cm}$ well defined cystic swelling in the retroareolar region was felt with normal overlying skin and nipple-areola complex. No axillary lymph nodes were palpable. FNAC of the lump showed features of infiltrating ductal carcinoma. Patient was taken up for modified radical mastectomy under general anaesthesia. Histopathological examination of the specimen revealed a cyst measuring $7 \times 4 \mathrm{~cm}$ containing hemorrhagic fluid. On gross examination, the cyst wall exhibited grey white papillary excrescences that protruded into the lumen. Histopathological diagnosis of intracystic papillary carcinoma was given. Being a rare entity, there is no definite guideline for the treatment of IPC; however, a surgical excision with a clear resection margin forms the mainstay of the treatment. These patients have very good outcome. The addition of hormonal treatment does not appear to have an impact on the patient outcome.
\end{abstract}

Keywords: Intra cystic, Papillary, Male, Breast carcinoma.

\section{Introduction}

Breast cancer is a relatively uncommon affliction in men, accounting for less than $1.5 \%$ of all the malignancies. Male patients account for less than $1 \%$ of all cases of breast cancers. Intra cystic papillary carcinoma (IPC) of the breast is known to account for $0.5 \%$ to $1 \%$ of all breast carcinoma. ${ }^{1,2}$ IPC represents a small subgroup of breast cancers and accounts 0.5-2\% of breast cancer in women. ${ }^{3}$ Some studies have suggested that they form a higher percentage in men, with an incidence range of 5-7.5\%. The prevalence of male breast cancer increases with age and is most commonly encountered in elderly patients with a mean age of 70 years. ${ }^{1,4,5}$ Furthermore, increased risk in men with gynecomastia has been reported. ${ }^{6}$

\section{Case Report}

A 61 year old gentleman presented with a painless, progressively enlarging lump in his left breast of 3 years duration. It was associated with serosanginous nipple discharge since 3 months. On examination, there was a single $8 \times 3 \mathrm{~cm}$ well defined cystic swelling in the retro-areolar region, with normal overlying skin and nipple-areola complex. There was no axillary lymphadenopathy. Ultrasonographic examination revealed a cystic lesion in the sub-areolar region with multiple mixed echoic lesions in the wall. FNAC was performed which yielded $15 \mathrm{ml}$ of haemorrhagic fluid. Centrifuged smears from the aspirated fluid showed malignant epithelial cells in singles and in small clusters along with many cyst macrophages (Fig. 1). A provisional diagnosis of infiltrating ductal carcinoma was made on FNAC. Tru-cut biopsy from the lesion was inconclusive as material obtained was scanty. Modified radical mastectomy was performed under general anaethesia without any postoperative complication.

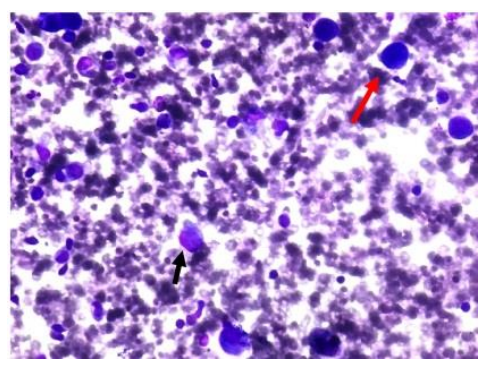

Fig. 1: Cytology showing dispersed malignant epithelial cells (black arrow and cyst macrophages (red arrow). (40X, Leishman)

\section{Gross Examination}

Cut section of specimen revealed a circumscribed cyst measuring $7 \times 4 \mathrm{~cm}$, located in the sub-areolar region containing hemorrhagic fluid. The cyst wall exhibited grey white papillary excrescences protruding into the lumen. Nipple and areola were unremarkable (Fig. 2) 


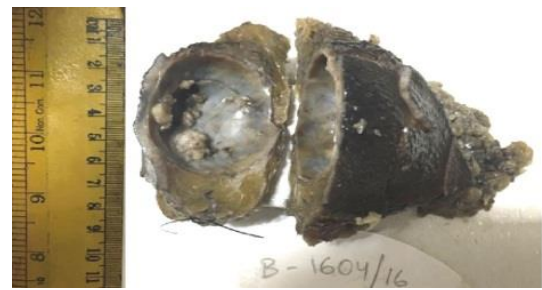

Fig. 2: Sub-areolar cyst showing papillary excrescenes

Microscopic Features: The tumour showed a papillary lesion composed of numerous delicate, branching, papillary fronds lined by epithelial cells showing stratification (Fig. 3). These cells exhibited moderate pleomorphism, hyper chromatic nuclei, and high nuclear cytoplasmic ratio (Fig. 4). There were 3-4 mitotic figures per HPF. There tumour was confined to cyst wall with no extra mural invasion. The nipple and all the surgical resected margins were free from tumour. All 13 lymph nodes dissected from the specimen were free of any metastasis from tumour deposits.

IHC: The tumour cells lining the papillary cores showed positive immunostaining for the estrogen receptor (ER) (Fig. 5) and progesterone receptor (PR) (Fig. 6). Smooth Muscle actin (SMA) positivity is seen in papillary cores (Fig. 7). The tumour cells stained negative for Her2/Neu overexpression (Fig. 8). Based on these findings, diagnosis of intracystic papillary carcinoma (Grade II) was made. Post operatively, patient did not receive any adjuvant chemotherapy or radio therapy. The patient is on regular follow up since eight months without any evidence of loco-regional or distant recurrences.

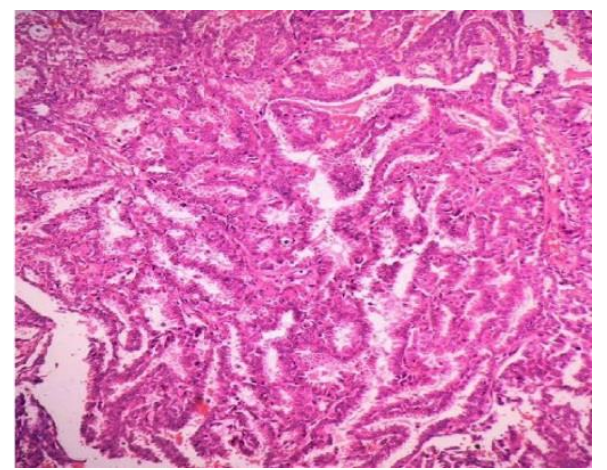

Fig. 3: Thin branching papillary fronds lined by tumor cells. (10X, H\&E)

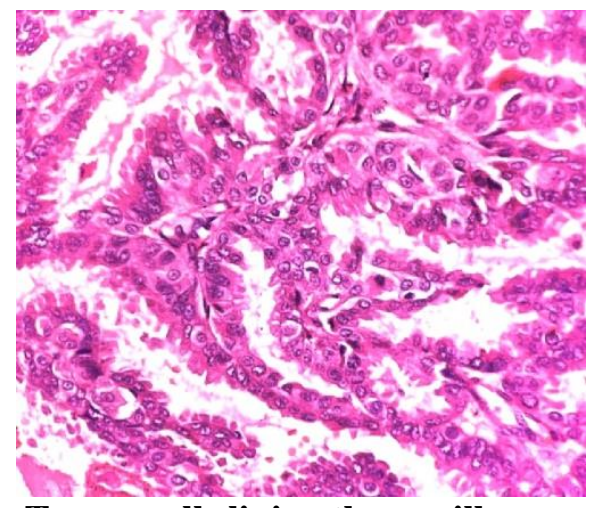

Fig. 4: Tumor cells lining the papillae

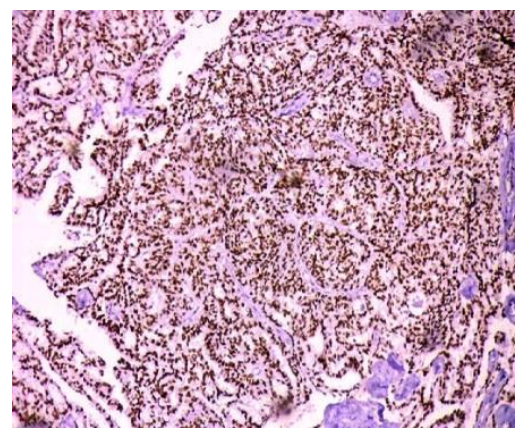

Fig. 5: ER positive tumor cells (10X)

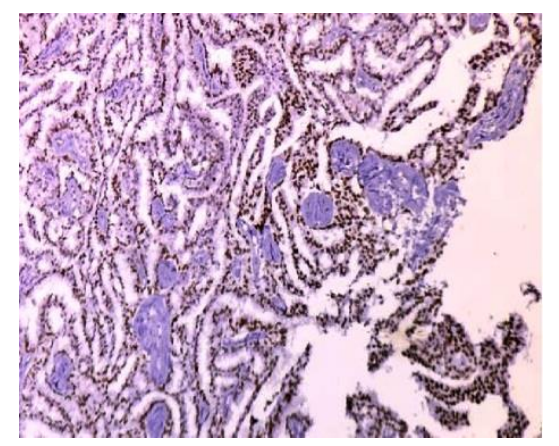

Fig. 6: PR positive tumor cells (10X)

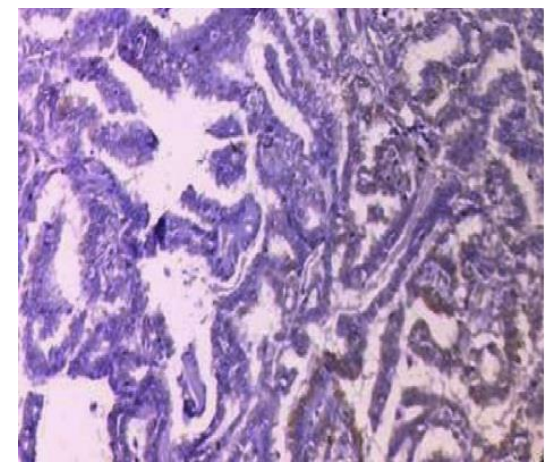

Fig. 7: SMA positivity in papillary cores $(40 \mathrm{X})$. No myoepithelial positivity seen 


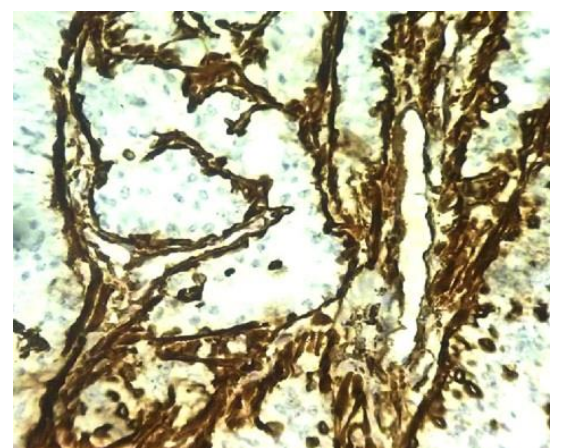

Fig. 8: Her2/Neu Negative in tumor cells (10X)

\section{Discussion}

Male breast cancer, a rare entity, has an incidence of one per 100,000 per annum.

Breast cancer in male patients represents $0.6 \%$ of all breast carcinomas and less than $1 \%$ of all malignancies in men. Male breast cancer shows racial predilection as it is more frequent in African Americans than in white Americans. The incidence of male breast cancer has a geographical variation with lower incidence rates seen in Japanese population as compared to the Chinese, African, Egyptian, and Jewish population. ${ }^{7}$ Intracystic papillary carcinoma comprises $2.6-5 \%$ of all male breast cancers. ${ }^{4,8}$

The exact etiology of male breast cancer is not known. However, risk factors implicated in male breast cancer include gynecomastia, radiation exposure, imbalance in estrogen-testosterone ratio, obesity, BRCA2 mutations. $^{9-12}$

Clinically, IPC present either as palpable lump in breast (75-90\%) or as nipple discharge. The lump is centrally located in $70-90 \%$ of the cases. In collective reviews, the disease has a slight predilection for the left breast same as is observed in this case report also.,13,14 IPC can be difficult to diagnose preoperative, hence a high clinical suspicion is required and triple assessment (i.e. clinical examination, radiological and histological assessment) is invaluable for establishing the diagnosis. ${ }^{15}$ Definitive diagnosis is usually made after excisional biopsy. ${ }^{16}$

Recent studies suggest that IPC should be considered as a low risk invasive carcinoma. ${ }^{17}$ Kraus and Neubecker established criteria to distinguish benign papilloma from the papillary carcinoma. ${ }^{18}$ Papillary carcinoma can be divided into the invasive and the noninvasive types. Subdivision of papillary carcinomas into 2 types was made by Carter et al. ${ }^{19}$ Recently, IPC has been divided into three types:

(i) Pure (with / without invasion),

(ii) With an associated intraductal carcinoma, and

(iii) With an associated, usual type of invasive ductal carcinoma.

Recently, Hill et al. used myoepithelial cell staining to determine the invasiveness of the tumour cells in papillary breast lesions, suggesting progression from an in- situ disease to an invasive disease. ${ }^{7}$ In men,infiltrating ductal carcinoma is the commonest histologic subtypes of invasive carcinoma (80\%), while papillary carcinoma, is seen in $5 \%$ of all the tumours. Lobular carcinoma is much less common in men than in women. There are four cellular patterns seen in IPC: cribriform, compact columnar epithelial, stratified spindle cell and a transitional cell form which resembles the urothelium. However, a combination of two or more of these patterns may also be seen. ${ }^{1}$ Regarding the regional and distant metastasis in IPC, studies report that axillary lymph node metastasis and distant metastasis of IPC occur in the range of $0 \%$ to $11 \%$ and $0 \%$ to $4 \%$ respectively. ${ }^{16}$ The oestrogen and progesterone receptor positivity is seen at higher rates in male breast cancer patients than in female breast cancer patients, but over-expression of HER2 and p53 mutation occur with equal frequency in men and women.

There is no definite guideline for the treatment of IPC, however, a surgical excision with a clear resection margin is the mainstay of the treatment. ${ }^{1,7}$ In male breast cancer patients, the stage at presentation and axillary node status are the most important prognostic indicators. DNA ploidy, EGFR expression, HER-2 oncogene expression, and ER status have not been shown to be predictive of disease-free survival. ${ }^{14}$ Fayanju et al. reviewed the adjuvant treatment given for IPC and found that patients with DCIS or microinvasive disease in association with IPC were more likely to receive radiotherapy and tamoxifen. ${ }^{20}$ The addition of radiation did not change the incidence of local recurrence or likelihood of death in these patients compared with those who did not receive radiation. ${ }^{2}$ Further, the addition of hormonal therapy does not appear to impact outcome in IPC, which has a good prognosis..$^{15}$ At 10 years, the survival rate of these patients remains $>95 \%$. Even in patients having invasion at the time of diagnosis, a relative survival rate of $>90 \%$ that of the general population at both 5 and 10 years is seen, supporting that there is low mortality for patients with IPC. ${ }^{21,22}$

\section{Conclusion}

IPC in males is a rare entity. Triple assessment is important in establishing the diagnosis. FNAC is often inaccurate and rarely diagnostic. Surgery is the cornerstone of treatment. The prognosis of IPC is excellent with low loco-regional and distant recurrence rates without hormonal treatment.

Acknowledgements: Head of Oncology, Dr.Neelesh Reddy for continuous follow up of case.

Prior Publication: None

Financial Support: Nil

Permission: College ethical committee 


\section{Conflicts of Interest: None}

\section{References}

1. Hariprasad S, Hariprasad P, Srinivas T. Intracystic Papillary Carcinoma of the Breast in Males: A Case Report and Review of the Literature. J ClinDiagn Res. 2013;7(3):568-70.

2. Kinoshita T, Fukutomi T, Iwamoto E, Takasugi M, Akashi-TanakaS, Hasegawa T. Intracystic papillary carcinoma of the breast ina male patient diagnosed by core needle biopsy: a case report. Breast. 2005;14(4):3224.

3. Czernobilsky B. Intracystic carcinoma of female breast. SurgGynecol Obstet. 1967;124:93-8.

4. Heller KS, Rosen PP, Schottenfeld D, Ashikari R, Kenne DW. Male breast cancer: clinicopathologic study of 97 cases. Ann Surg. 1978;188:60-5.

5. Wolff M, Renis MS. Breast cancer in males: clinicopathologic study of 40 patients. New York: Masson Publishing; 1981.

6. Liechty RD, Davis J, Gleysteen J. Cancer of the male breast: forty cases. Cancer. 1967;20:1617-24.

7. Romics L, O'Brien ME, Relihan N, O'Connell F, Redmond HP.Intracystic papillary carcinoma in a male as a rare presentation of breast cancer: a case report and literature review. J Med Case Rep.2009;3:13-17.

8. Giordano SH, Cohen DS, Buzdar AU, et al. Breast carcinoma in men: a population based study. Cancer. 2004;101:51-7.

9. Sasco AJ, Lowenfels AB, Pasker-de Jong P. Review article: epidemiology of male breast cancer. A metaanalysis of published case-control studies and discussion of selected aetiological factors. Int J Cancer. 1993;53:538-49.

10. Shah P, Robbani I, Shah O. Clinicopathological study of male breast carcinoma: 24 years of experience. Ann Saudi Med. 2009;29:288-93.

11. Kwiatkowska E, Teresiak M, Filas V, et al. BRCA2 mutations and androgen receptor expression as independent predictors of outcome of male breast cancer patients. Clin Cancer Res. 2003;9:4452-9.

12. Ruddy KJ, Winer EP. Male breast cancer: risk factors, biology, diagnosis, treatment, and survivorship. Ann Oncol. 2013;24:1434-43.

13. Hu ZI, Liu C, Fisher PC, Cohen JA. Intracystic papillary carcinoma of the breast in a male patient. Rare Tumors. 2016; 8:6050:8-10.

14. Pant I, Joshi SC. Invasive papillary carcinoma of the male breast: Report of a rare case and review of the literature. J Cancer Res Ther. 2009;5(3):216-8.

15. Stamatakos M, Stefanaki C, Stasinou T, Papantoni E, Alexiou I, Kontzoglou K. Intracystic Papillary Carcinoma of the Breast in Males. In Search of the Optimal Treatment for this Rare Disease. Breast Care. 2011. 6(5):399-40.

16. Solorzano CC, Middleton LP, Hunt KK, Mirza N, Meric F, Kuerer HM, et al. Treatment and outcome of patients with intracystic papillary carcinoma of the breast. Am J Surg. 2002;184:364-68.

17. Lee SK, Choi JH, Lim HI, Kim WW, Kim S, Cho EY et al. Experience with Intracystic Papillary Carcinoma of the Breast at a Single Institute in Korea. J Breast Cancer. 2009;12:338-43.

18. Kraus FT, Neubecker RD. The differential diagnosis of papillary tumors of the breast. Cancer. 1962;15:444-55.

19. Fisher ER, Palekar AS, Redmond C, Barton B, Fisher B. Pathologic findings from the National Surgical Adjuvant
Breast Project (protocol no. 4).VI. Invasive papillary cancer. Am J ClinPathol. 1980;73:313-22.

20. Fayanju OM, Ritter J, Gillanders WE, Eberlein TJ, Dietz JR, Aft R et al. Therapeutic management of intracystic papillary carcinoma of the breast: the roles of radiation and endocrine therapy. Am J Surg. 2007;194(4):497-500.

21. Carter D, Orr SL, Merino MJ. Intracystic papillary carcinoma of the breast. After mastectomy, radiotherapy or excisional biopsy alone. Cancer. 1983;52:14-19.

22. Harris KP, Faliakou EC, Exon DJ, et al. Treatment and outcome of intracystic papillary carcinoma of the breast. Br J Surg. 1999;86:1274. 